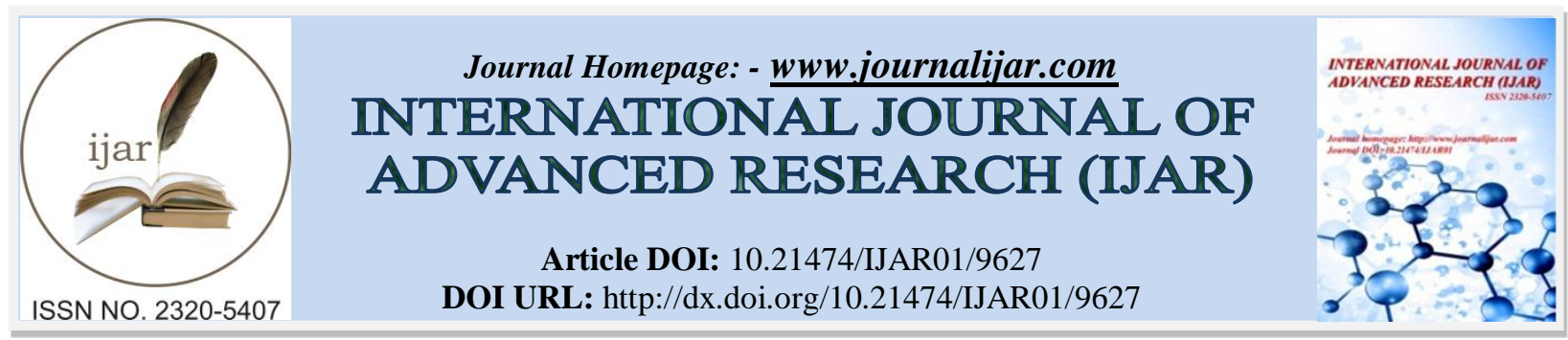

RESEARCH ARTICLE

\title{
GROSSESSE EXTRA-UTÉRINE CORNUALE ET INTRA-MURALE: À PROPOS DE DEUX CAS AVEC REVUES DE LA LITTÉRATURE. CORNUAL AND INTRA-MURAL PREGNANCY : ABOUT TWO CASES AND LITERATURE REVIEWS.
}

\section{Moulay El Mehdi El Hassani ${ }^{1}$, Saad Benali ${ }^{2}$, Jihad Drissi ${ }^{2}$, Jaouad Kouach ${ }^{2}$ and Moulay Driss Moussaoui Rahali $^{2}$.}

1. Faculté de Médecine et de Pharmacie de Fès.

2. Service de Gynécologie-Obstétrique, Hôpital Militaire d'Instruction Mohamed V, Rabat, Maroc.

\section{Manuscript Info}

………................

Manuscript History

Received: 02 July 2019

Final Accepted: 04 August 2019

Published: September 2019

\section{Abstract}

The cornual pregnancy is a rare entity that represents almost $2 \%$ of ectopic pregnancies whereas the intramural pregnancy is the rarest one. The diagnosis is almost always based on laparoscopy. The risk of rupture with severe bleeding remains high especially when the diagnosis is late. The treatment is often surgical, though in some cases, medical treatment by methotrexate has been described. The objective of this work is to describe two rare forms of ectopic pregnancies and expose its diagnostic features, management and prognosis.

Authors report two cases of cornual and intra-mural pregnancy, the first one about a 27 year-old woman without risk factors, the second one about a woman with history of abortion.

Copy Right, IJAR, 2019,. All rights reserved.

\section{Introduction:}

Les grossesses extra-utérines sont une cause redoutée de métrorragies du premier trimestre. Elles correspondent à la nidation et au développement de l'œuf en dehors de la cavité utérine.

La grossesse cornuale; implantation de l'œuf dans la corne utérine, est une entité rare qui représente près de $2 \%$ des grossesses extra-utérines [1].

La localisation intra-murale est rare et correspond à la nidation de l'œuf dans la profondeur du myomètre au sein d'une cavité ne communiquant ni avec la cavité utérine ni avec la lumière tubaire [2].

\section{Observation 1:}

Nous rapportons le cas d'une grossesse cornuale découverte en per-opératoire chez une patiente de 27 ans, deuxième geste, primipare, mère d'un enfant vivant, n'ayant pas de facteurs de risque de grossesse extra-utérine. Grossesse actuelle spontanée, estimée à 11 semaines d'aménorrhée (grossesse retenue sur le test qualitatif de grossesse). La patiente a été admise pour algies pelviennes aigües avec métrorragies du premier trimestre avec à l'examen clinique une défense pelvienne latéralisée à droite et des signes d'irritation péritonéale (cris du Douglas). Par ailleurs, la patiente était stable sur le plan hémodynamique.

Corresponding Author: Moulay El Mehdi El Hassani. droite

Address: Faculté de médecine et de pharmacie de Fès. 
de $2.5 \mathrm{~cm}$ échogène, hétérogène et un épanchement péritonéal de moyenne abondance au niveau du cul de sac de Douglas et en inter-vésico-utérin. Taux de béta-Hcg à 2000. Devant la forte suspicion de grossesse extra-utérine, la patiente a été admise immédiatement au bloc opératoire avec demande de sang et bilan de crase. Une minilaparotomie a été faite avec à l'exploration:

1. Un hémopéritoine de moyenne abondance: $200 \mathrm{cc}$ aspiré.

2. Une grossesse cornuale droite de $2 \mathrm{~cm}$ rompue (Fig.1).

3. Une corne contro-latérale de bonne qualité.

4. Absence d'adhérences ou d'endométriose pelvienne.

Une extériorisation de la GEU avec résection cornuale a été réalisée (Fig.2). Les suites opératoires étaient simples. Taux d'Hcg après $48 \mathrm{~h}$ inférieur à $25 \%$ du taux initial.

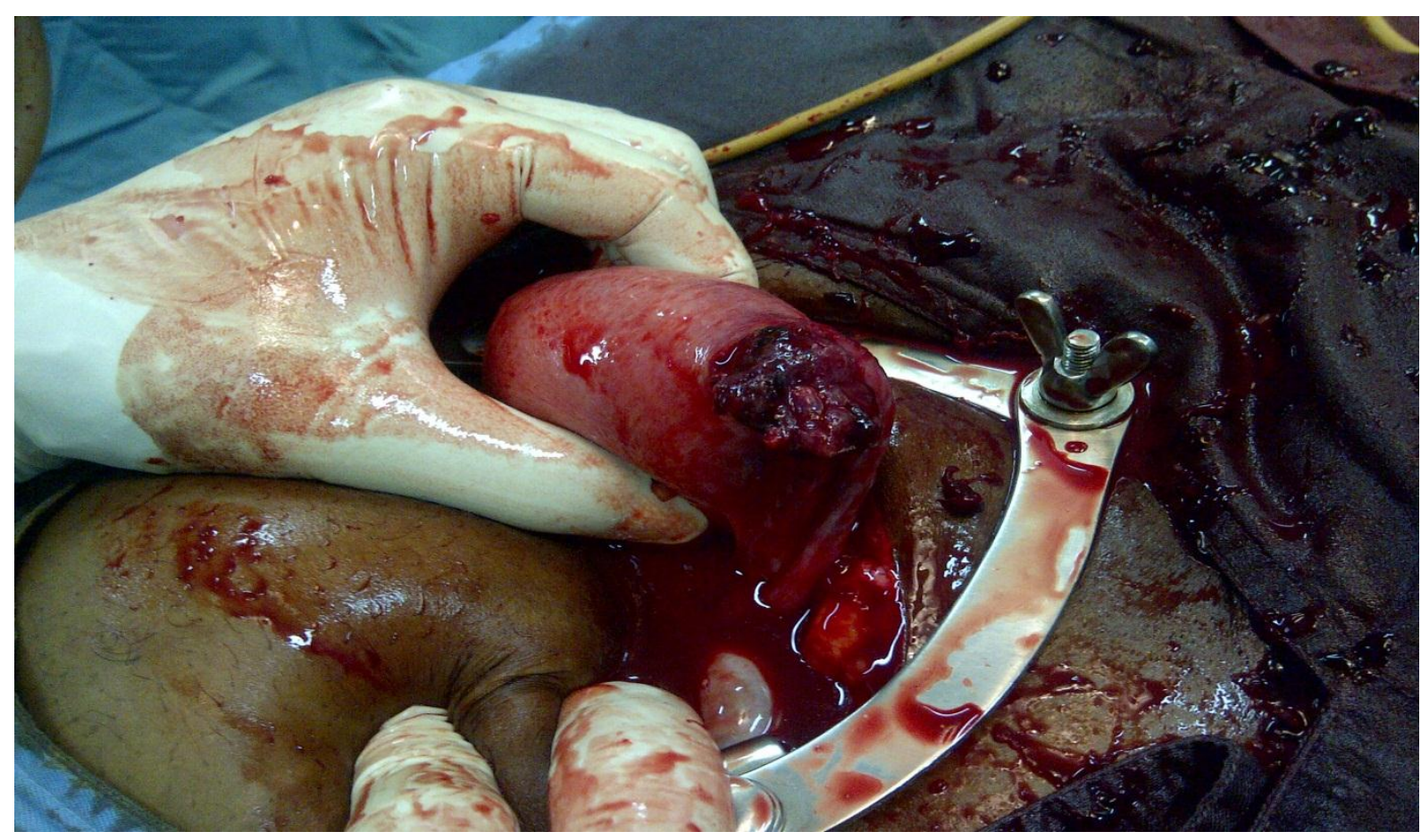




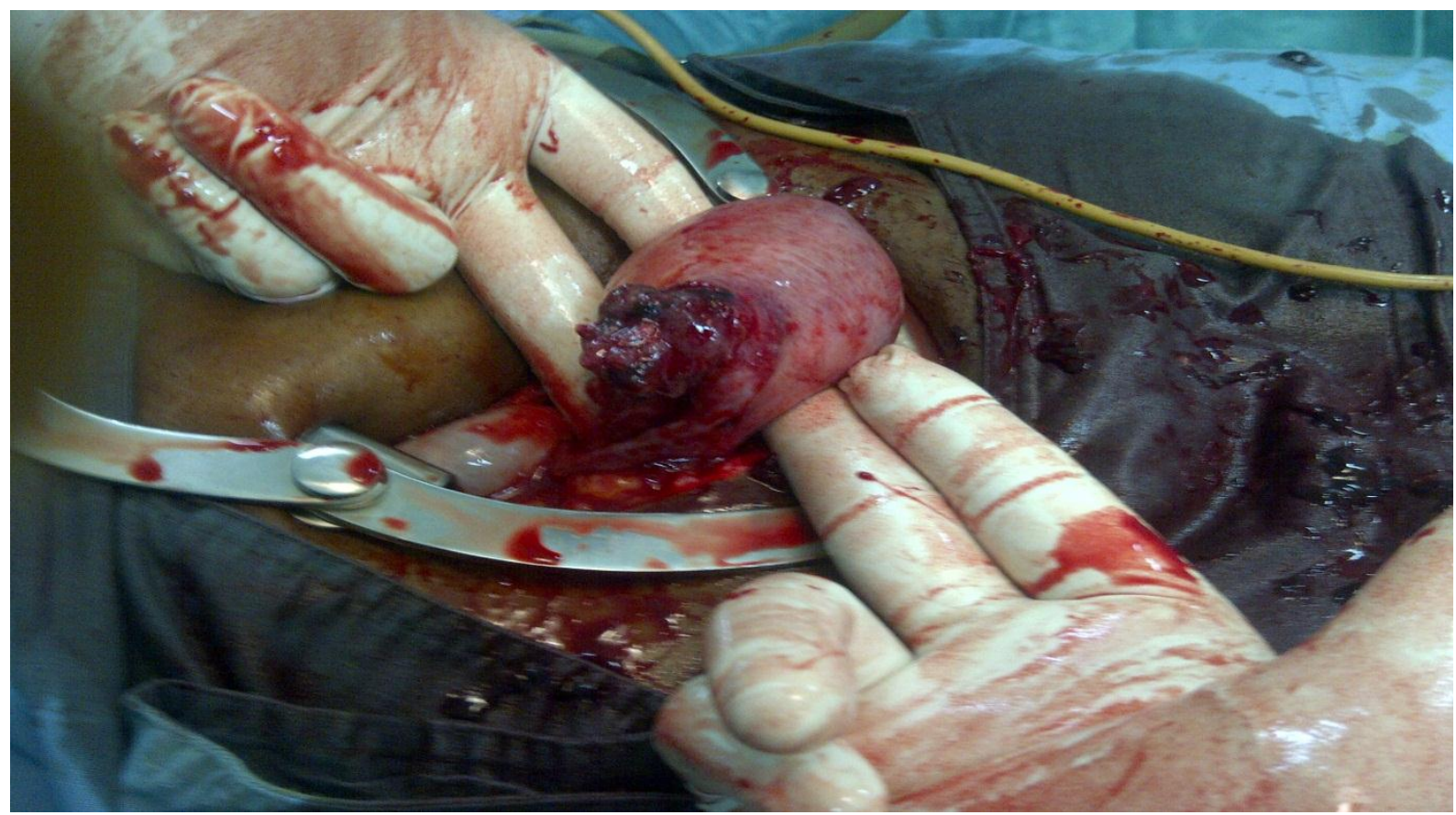

Fig 1: Grossesse cornuale droite: aspect per-opératoire

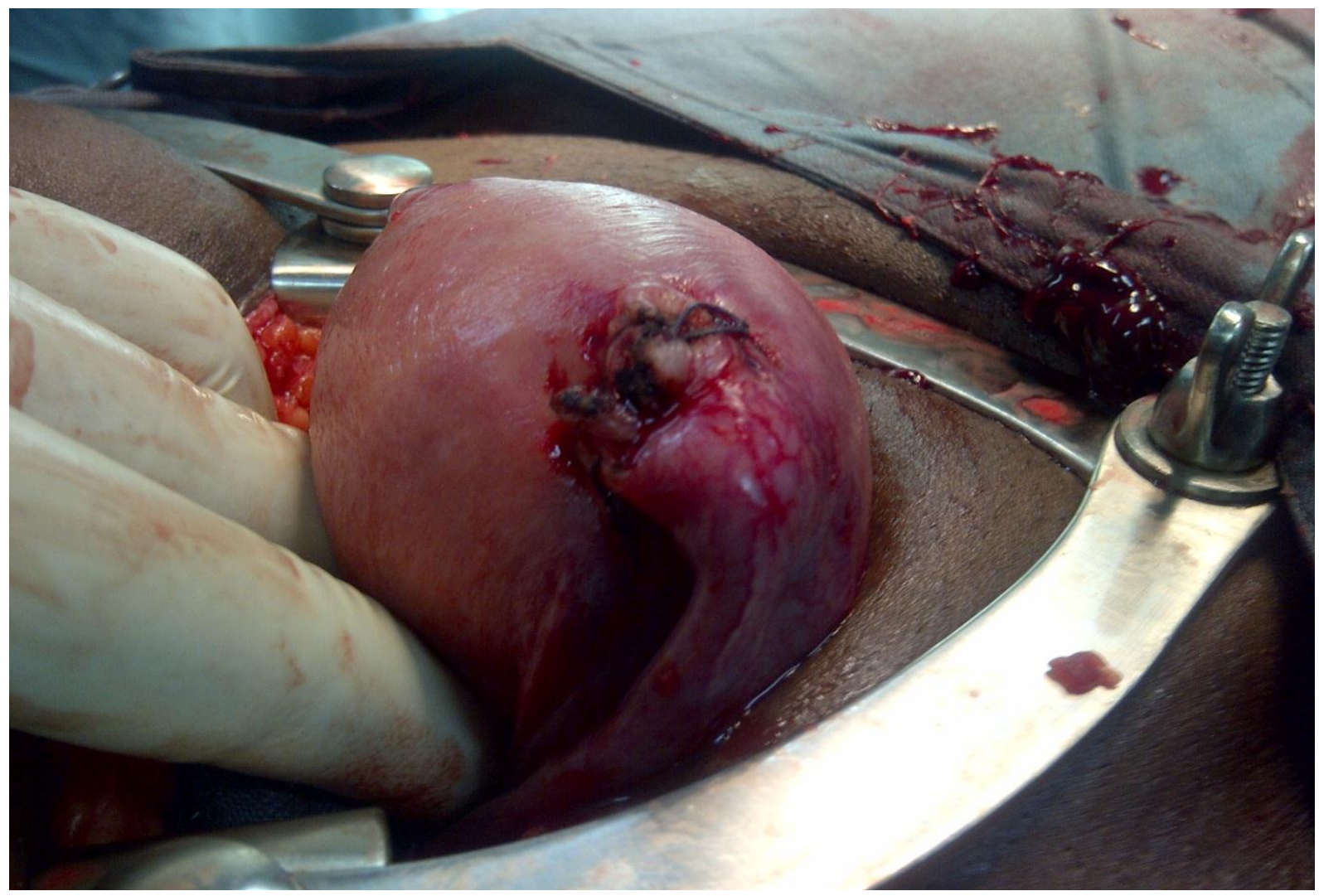

Fig 2: Résultat après cure chirurgicale

\section{Observation 2:}

Il s'agit d'une patiente âgée de 32 ans nullipare chez qui, une échographie pelvienne a était réalisée pour des douleurs pelviennes sans métrorragies sur une aménorrhée de deux mois. L'interrogatoire retrouve un antécédent de fausse 
couche provoquée par curetage il y a 3 ans. L'échographie pelvienne par voie sus pubienne a permis de poser le diagnostic de grossesse ectopique intra-murale de 6SA+4 jours avec un embryon vivant (Fig.3). Au décours de l'examen échographique, la survenue de métrorragies abondantes faites de sang rouge vif imposa une laparotomie en urgence. Le diagnostic de grossesse intra murale compliquée de rupture utérine a été posé en per-opératoire. Une hystérectomie fut réalisée avec des suites opératoires simples.

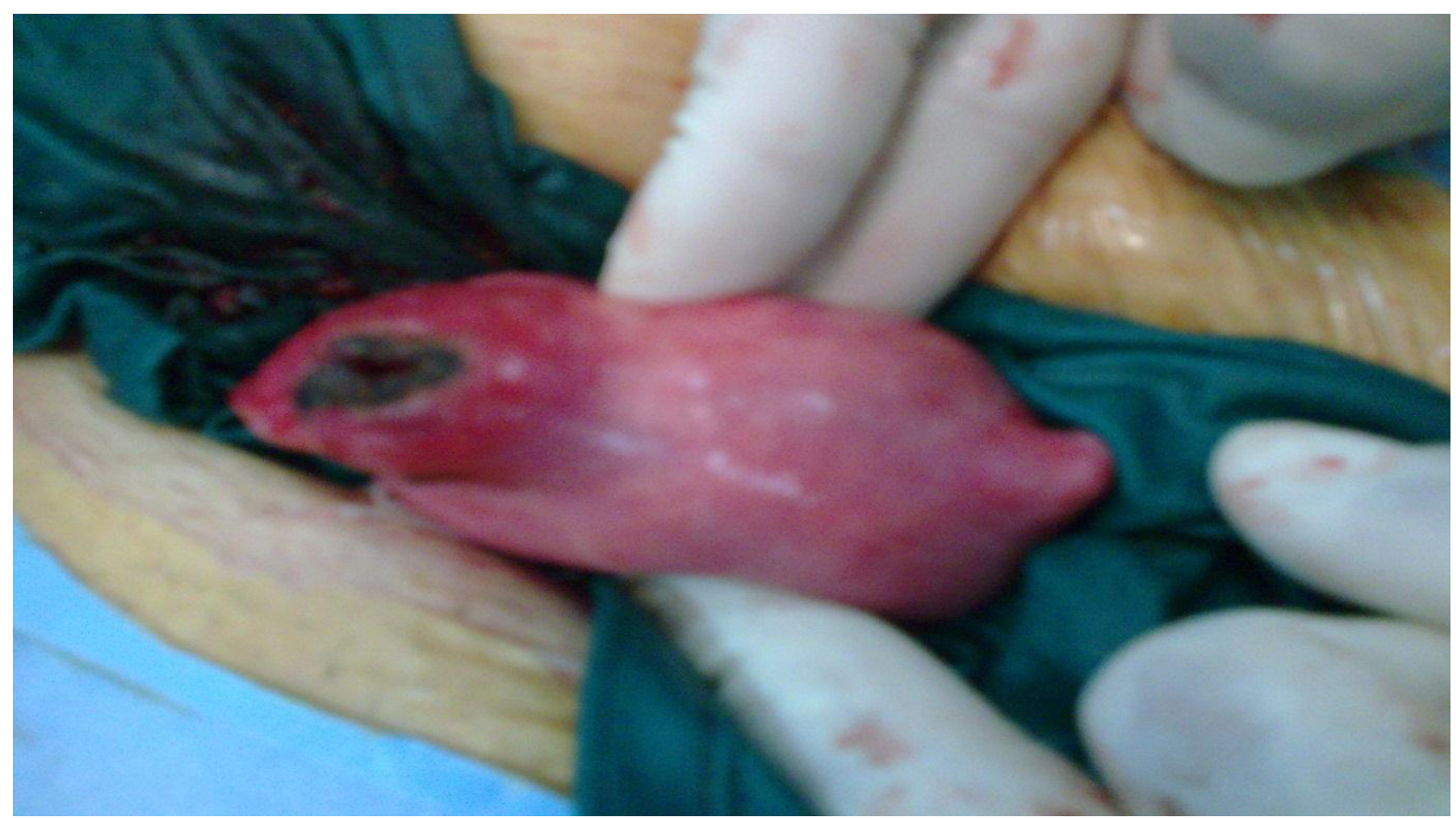

Fig 3: Grossesse intra-murale: aspect per-opératoire

\section{Discussion:- \\ 1-Grossesse cornuale: \\ Définition}

La grossesse cornuale est une entité rare qui se distingue des autres types de grossesse extra-utérine par son plus grand risque hémorragique avec une indication plus large de traitement radical.

\section{Facteurs de risque}

Outre les facteurs de risque communs des grossesses extra-utérines (âge maternel avancé, tabac, DIU, contraception par microprogestatifs, endométriose et antécédents de chirurgie pelvienne, infections génitales hautes récidivantes), les facteurs spécifiques de la localisation cornuale sont: les malformations utérines avec corne rudimentaire et les antécédents de salpingectomie avec grossesse cornuale sur le moignon utérin [3].

\section{Diagnostic}

1. La clinique est similaire aux autres localisations tubo-ovariennes: algies pelviennes aigües, métrorragies du premier trimestre, choc hémorragique.

2. Béta-Hcg: positive.

3. Echographie: le diagnostic de grossesse cornuale est souvent pris à défaut par l'échographie sus-pubienne. En effet, le sac gestationnel peut paraitre fundique intra-utérin retardant ainsi le diagnostic au stade de rupture engageant le pronostic vital de la femme [4]. Dans sa forme typique, à l'échographie endovaginale, l'utérus parait vide, le sac gestationnel quand il est visible réalise une masse fundique cessile entourée du myomètre.

4. Cependant le siège cornual est souvent découvert en per-opératoire.

\section{Prise en charge}

Le traitement radical par salpingectomie avec résection cornuale est le traitement classique [3]. Le traitement médical par méthotrexate in situ semble être une alternative thérapeutique intéressante quand elle est possible [5]. L'embolisation sélective a été récemment proposée comme traitement efficace [6]. 


\section{Pronostic}

Le pronostic de fertilité et le risque de récidive dépendent bien évidemment de l'état de la trompe contro-latérale. Quant au pronostic obstétrical, il est marqué par le risque de rupture utérine, ainsi, la césarienne semble être justifiée lors d'une grossesse ultérieure [5].

\section{2-Grossesse intra-murale:}

La grossesse intra murale est une entité extrêmement rare représentant moins de $1 \%$ des grossesses extra-utérines [7]. Jusqu'en 2013, seuls 53 cas ont été rapportés dans la littérature. Ces grossesses surviennent en cas de cicatrices myométriales communicant avec la cavité utérine. Il peut s'agir de cicatrices de césarienne ou de myomectomie, de cavités adénomyomateuses ou après curetage comme dans notre cas [2]. Le principal risque de cette localisation est la rupture utérine avec hémorragie cataclysmique [8]. Cliniquement elle se traduit avant la survenue de la rupture par l'association d'une aménorrhée, de douleurs pelviennes, de métrorragies et d'un taux de B-Hcg positif. Ces signes sont ceux de toute grossesse ectopique ou d'un avortement spontané. Cette absence de spécificité des signes cliniques fait tout l'intérêt de l'imagerie. L'échographie pose le diagnostic en visualisant un sac ovulaire excentré et une cavité utérine vide ou siège d'une hématométrie. Selon Luo et al. Il existerait trois formes échographiques de grossesse intra murale: la forme kystique, la forme nodulaire et la forme rompue [9]. Le diagnostic reste difficile à l'échographie, la mise évidence de l'embryon permet d'écarter les classiques diagnostics différentiels que sont un myome en transformation kystique et une tumeur trophoblastique. Le Doppler couleur, en visualisant l'hypervascularisation et les reconstructions 3D en permettant une meilleure localisation du sac améliorent le diagnostic échographique. L'IRM est d'un apport certain en cas de doute diagnostic [8]. Le traitement est le plus souvent chirurgical, parfois conservateur par laparotomie [8] ou laparoscopie [10]. Dans les cas vus tôt un traitement médical par injection de méthothrexate donne de bons résultats [7].

\section{Conclusion:-}

La grossesse interstitielle et intra-murale sont des grossesses ectopiques rares, de diagnostic difficile qui peuvent mettre en jeu le pronostic vital ainsi que la fertilité maternelle. La prise en charge classique est chirurgicale. Lors des grossesses ultérieures le clinicien se méfiera des risques de récidive et de rupture utérine.

\section{Conflits d'intérêts:}

Les auteurs ne déclarent aucun conflit d'intérêts.

\section{Références:-}

1. B. Blanc, C. Jamin, C. Sultan. Traité de gynécologie médicale. Springer Amazon. p.305.

2. Kirk E, McDonald K, Rees J, Govind A. Intramural ectopic pregnancy: a case and review of the literature. Eur J Obstet Gynecol Reprod Biol. 2013;168(2):129-33.

3. X. Douysset, E. Verspyck, A. Diguet, L. Marpeau, I.Chanauaz- Lachay, B.Resch, P. Sergent. Grossesse interstitielle : traitement chirurgical, fertilité et devenir obstétrical : l'expérience rouennaise. Gynécologieobstétrique et fertilité 2013.

4. N. Kouame, A. N’Doumoua, R. N'Gbesso, A. Keita. Grossesse cornuale : une entité rare de grossesse extrautérine. Imagerie de la femme-Vol. 21-N.2- p68-71.

5. O. Jourdain, M.Fontanges, A. Schiano, F. Rauch, J-M. Gonnet. Prise en charge des autres ectopies annexielles (cornuales, interstitielle, angulaire, ovarienne). Journal de Gynécologie Obstétrique et biologie de la Reproduction-Vol. 32-N.S7- p93-100.

6. P. Deruelle, E. Closset, C. Lions, J.-P. Lucot. Conservation de la fertilité après embolisation sélective d'une grossesse cornuale. Gynécologie Obstétrique \& Fertilité, Volume 34, Issue 10, October 2006, Pages $914-916$

7. Ong C, Su L-L, Chia D, Choolani M, Biswas A. Sonographic diagnosis and successful medical management of an intramural ectopic pregnancy. J Clin ultrasound. 2010;38(6):320- 4

8. Fadhlaoui A, Kharouf M, Nouira K, Chaker A, Zhioua F. Ruptured intramural pregnancy with myometrial invasion treated conservatively. Case Rep Obstet Gynecol. 2011;2011:965910

9. Luo Z, Zhou P, Gao F, HE W, Luo S. Diagnosis of intramural pregnancy by endoluminal color Doppler ultrasonography and review of the literature. J South Med Univ. 2010;30(10):2343- 6

10. Nabeshima H, Nishimoto M, Utsunomiya H, Arai M, Ugajin T, Terada Y et al. Total laparoscopic conservative surgery for an intramural ectopic pregnancy. Diagn Ther Endosc. 2010;2010:504062 